Article

\title{
Synthesis and Application in Polypropylene of a Novel of Phosphorus-Containing Intumescent Flame Retardant
}

\author{
Jian-Dong Zuo ${ }^{1, *}$, Shu-Mei Liu ${ }^{2}$ and Qi Sheng ${ }^{2}$
}

1 Shenzhen Key Laboratory of Special Functional Materials, College of Materials Science and Engineering, Shenzhen University, 518060 Shenzhen, China

2 College of Materials Science and Engineering, South China University of Technology, 510640, Guangzhou, China; E-Mails: Shumeiliu@163.com (S.L.); mshengqi@yahoo.com.cn (Q.S.)

* Author to whom correspondence should be addressed; E-Mail: qingfeng_9709@126.com. Tel: +86-755-26535133; Fax: +86-755-26536239.

Received: 17 September 2010 / Accepted: 13 October 2010 / Published: 28 October 2010

\begin{abstract}
A novel phosphorus-containing triazine oligomer poly(2-morpholinyl-4-pentaerythritol phosphate-1,3,5-triazine) (PMPT) was synthesized as a kind of tri-component intumescent flame retardant (IFR). The chemical structure of PMPT was characterized by FTIR, ${ }^{1} \mathrm{H}-\mathrm{NMR}$ and ${ }^{31} \mathrm{P}-\mathrm{NMR}$, and the mechanical and flammability properties of FR-PP were measured. The FTIR results showed that the expected chemical reactions had happened at each step. The ${ }^{1} \mathrm{H}-\mathrm{NMR}$ and ${ }^{31} \mathrm{P}-\mathrm{NMR}$ spectra also agreed with the chemical structure of PMPT. The slight effect of PMPT on the mechanical properties of FR-PP suggested that PMPT and PP are compatible. The high limited oxygen index (LOI) values of FR-PP revealed that PMPT was an efficient IFR and there was the synergistic effect between PMPT and ammonium polyphosphate/ pentaerythritol (APP/PER).
\end{abstract}

Keywords: triazine oligomer PMPT; intumescent flame retardant; phosphate-containing

\section{Introduction}

Intumescent Flame Retardant (IFR) systems have been used recently in flame retarded Polypropylene (FR-PP) as a kind of halogen-free flame retardant. Intumescent flame retardant compounds are usually composed of an acid source, a carbon source and a gas source, such as ammonium polyphosphate (APP), pentaerythritol (PER) and melamine [1]. However, the larger dosage 
of IFR compound needed and the poor compatibility with the polymer always impair the mechanical properties of PP.

A tri-component IFR is a new method which can reduce the amount of flame retardant and improve the thermal stability of the resulting FR-PP [2 3]. The main component of an intumenscent flame retardant is the element nitrogen, which has a good synergic flaming effect with phosphorus in the flame retarding [4-5]. It was found that triazines and their derivatives are good charring agents because of their abundant nitrogen and tertiary nitrogen structure [6-7]. Liu synthesized the phosphoruscontaining triazine oligomer poly(2-piperazinylene-4-morpholino-1,3,5-triazine) (PPMT) and found that PPMT had a good effect in the delaying combustion of PP [8], but PPMT has no carbon atoms in the main molecular chain, so it has no carbon source and has to be used together with PER in order to achieve good flame retarding. In this paper, a novel phosphorus-containing triazine oligomer poly(2morpholinyl-4-pentaerythritol phosphate-1,3,5-triazine) (PMPT) was synthesized and the chemical structure of the product was character by means of FTIR, ${ }^{1} \mathrm{H}-\mathrm{NMR}$ and ${ }^{31} \mathrm{P}-\mathrm{NMR}$.

\section{Results and Discussion}

\subsection{Reaction mechanisms (Scheme 1)}

In tricyanogen chloride the stronger electron withdrawing group is the chlorine atom, which is a Lewis acid. There are lone pair electrons on the triethyl phosphate, which is a Lewis base. The reaction of tricyanogen chloride and triethyl phosphate can thus be regarded as a simple $\mathrm{SN}_{2}$ Lewis acid-base reaction. The net result is that the chlorine atom is easy to remove from the carbon atom and the yield of the acid-base reaction was improved

Scheme 1. Reaction mechanism of PMPT.

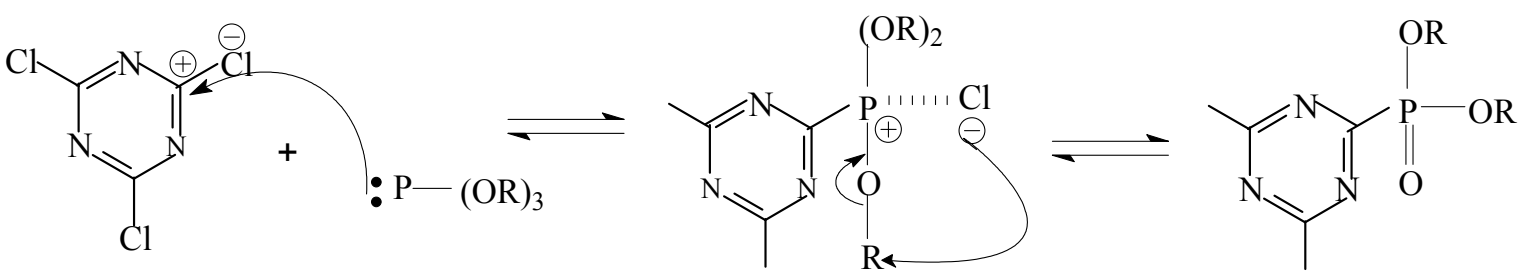

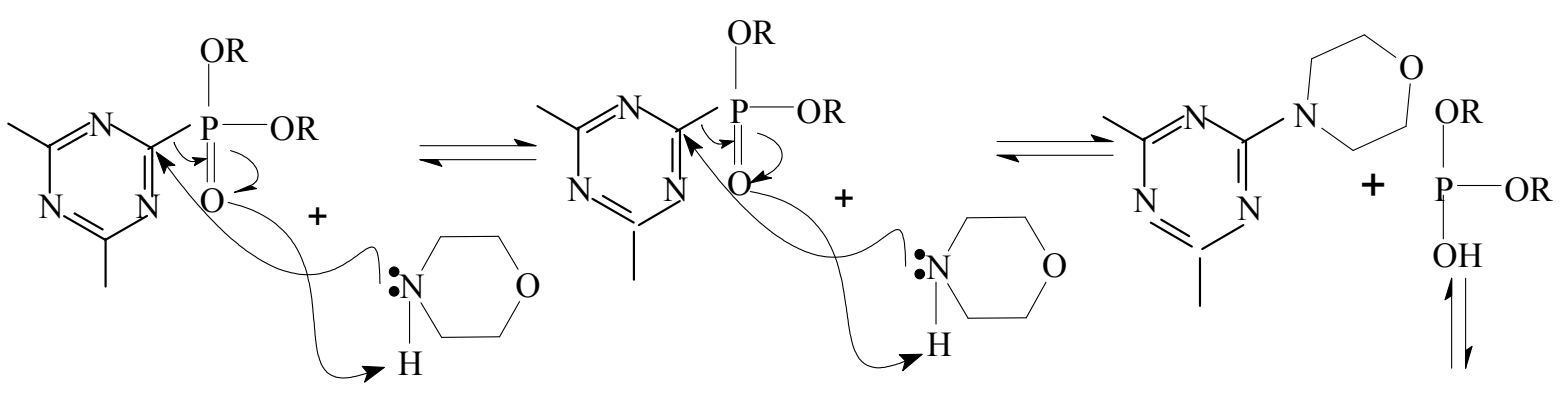<smiles>[R]O[PH]([R])=O</smiles> 
In the second step the alkalinity of morpholine is greater than that of triethyl phosphate and the $\mathrm{N}$ atom has the stronger nucleophilicity on the morpholine, so it can attack the carbon atom with the positive charge close to the chlorine atom. The second reaction is therefore a nucleophilic substitution that can happen at room temperature. The reaction of compound $\mathbf{2}$ and pentaerythritol is a trasesterification. Dibutyl tin oxide as a neutral catalyst has no effect on the properties of the product and there is no need to separate it from the solution. Alcohol was continually evaporated from the solution in order to improve the yield.

\subsection{FTIR of PMPT}

Figures 1, 2 and 3 show the FTIR spectra of compound 1, compound 2 and PMPT, respectively. There are some specific absorption peaks for compound 1. The bands at $2,986 \mathrm{~cm}^{-1}$ and $2,936 \mathrm{~cm}^{-1}$ were attributed to the stretching vibration of $\mathrm{C}-\mathrm{H}$ in $-\mathrm{CH}_{2}$ and $-\mathrm{CH}_{3}$. The absorption at $1,499 \mathrm{~cm}^{-1}$ was assigned to the skeleton vibration of $\mathrm{C}=\mathrm{N}$ in the triazine ring [9]. It is possible that the vibrational absorption of the triazine ring skeleton at $1,557 \mathrm{~cm}^{-1}$ shifts to the low frequency influenced by $\mathrm{P}=\mathrm{O}$ group [10]. The band at $1,261 \mathrm{~cm}^{-1}$ is attributed to the stretching vibration of the $\mathrm{P}=\mathrm{O}$ group, indicating that the expected chemical reaction between triethyl phosphite and tricyanogen chloride has indeed taken place. In Figure 2, the absorption signal at $1116 \mathrm{~cm}^{-1}$ was attributed to the asymmetric stretching vibration of the ether bond in the morpholinyl ring. The stretching vibration of $\mathrm{P}=\mathrm{O}$ was slightly shifted to a lower frequency $\left(1,254 \mathrm{~cm}^{-1}\right)$.

Figure 1. FTIR spectrum of compound 1.

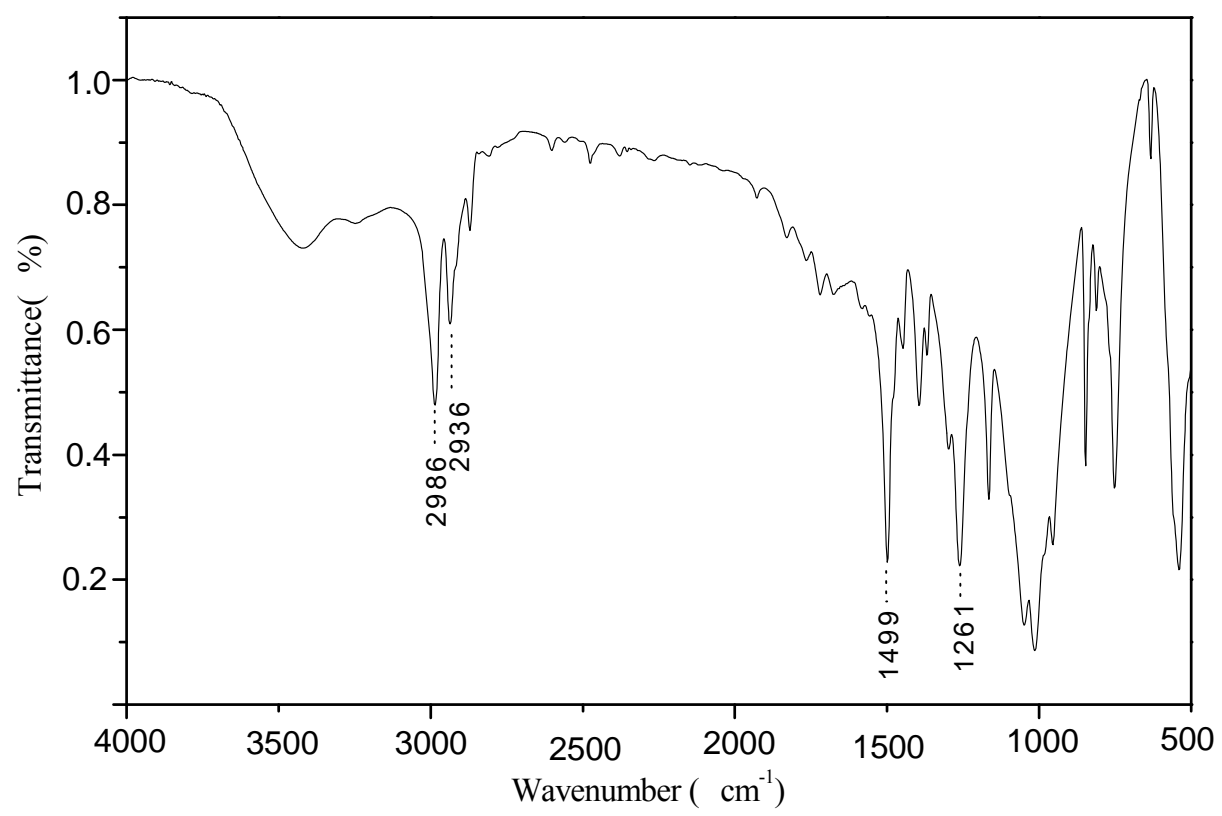

In Figure 3, the absorption peak at $1,267 \mathrm{~cm}^{-1}$ still be assigned to the stretching vibration of the $\mathrm{P}=\mathrm{O}$ group. The absorption peak of tertiary carbon bond can't be clearly observed because of the overlap with the $\mathrm{P}=\mathrm{O}$ group at about $1,245 \sim 1,180 \mathrm{~cm}^{-1}$ [11]. The appearance of the three $\mathrm{P}-\mathrm{O}$ group absorption bands at $1,036 \mathrm{~cm}^{-1}$ showed that the phosphor-containing triazine oligomer had been 
generated. The strong band at 3,394 $\mathrm{cm}^{-1}$ was the absorption peak of the hydroxyl group. This ocurrs when pentaerythritol is the terminal group and the oligomer has the stronger water absorption.

Figure 2. FTIR spectrum of compound 2.

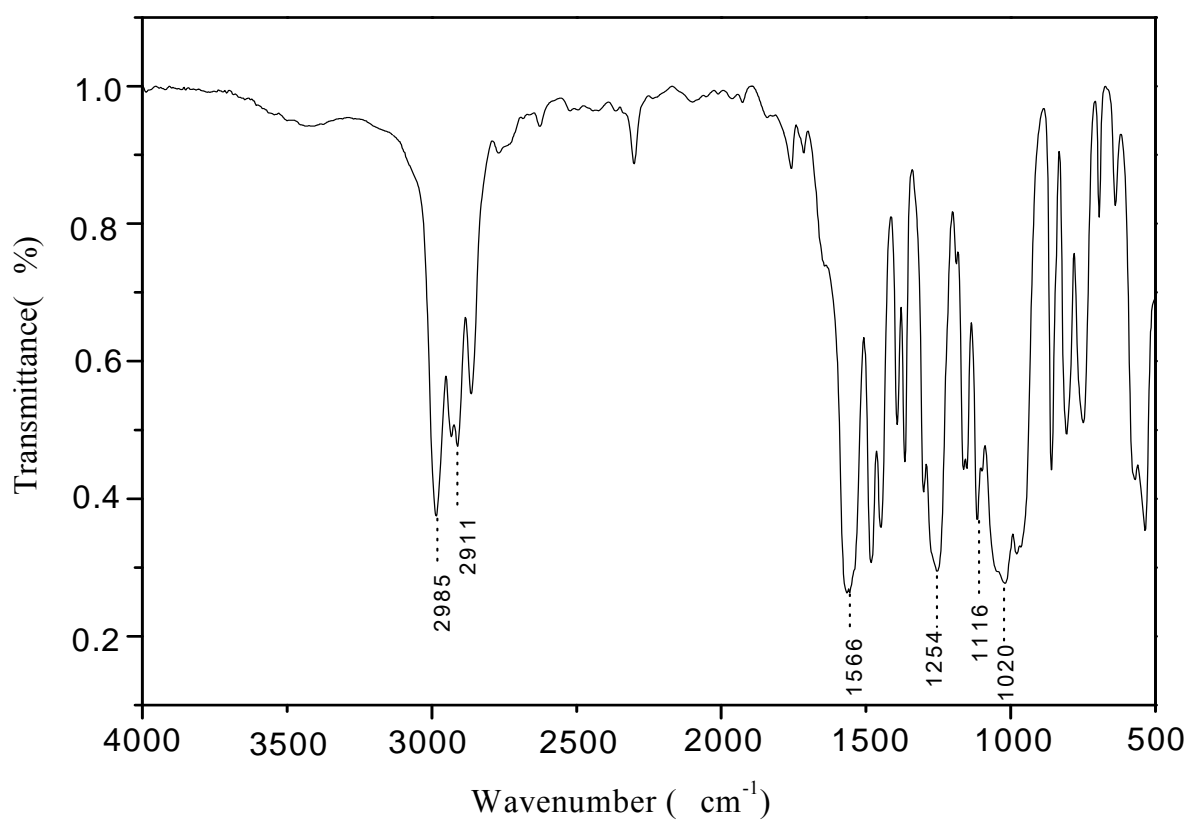

Figure 3. FTIR spectrum of phosphorus-containing triazine oligomer PMPT.

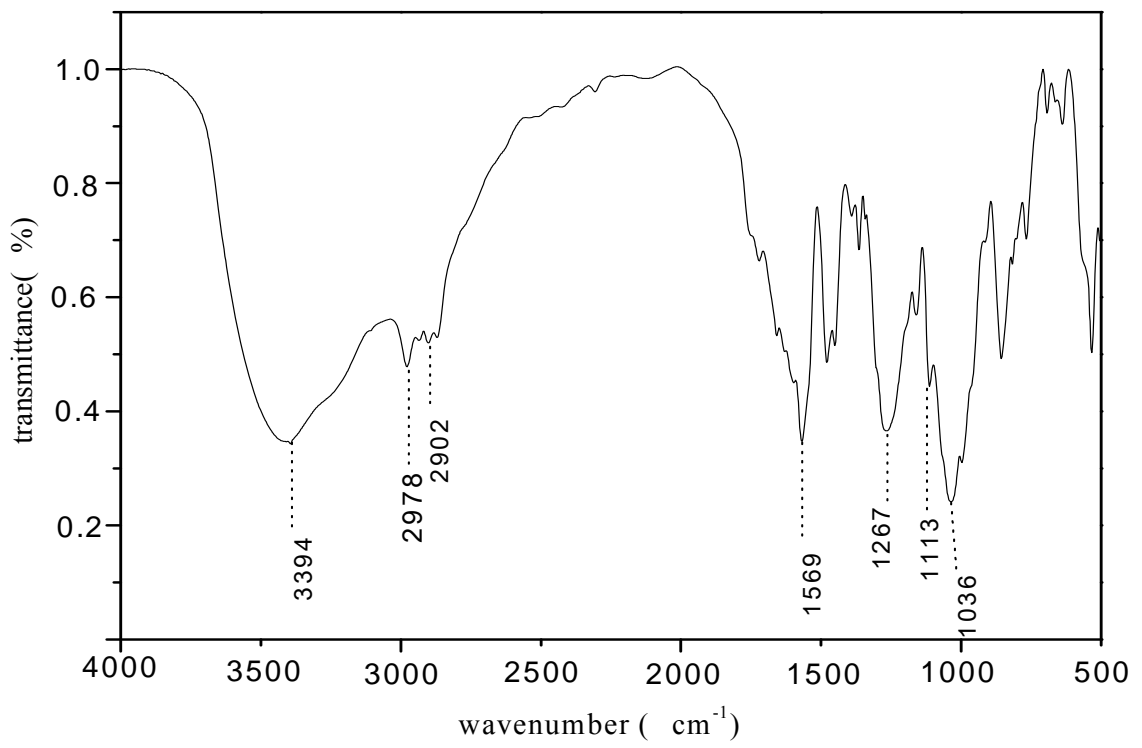

\section{2. ${ }^{1} \mathrm{H}-\mathrm{NMR}$ of $\mathrm{PMPT}$}

The ${ }^{1} \mathrm{H}-\mathrm{NMR}$ spectrum of PMPT is shown in Figure 4. The strong peak at $\delta=4.75 \mathrm{ppm}$ comes from the $\mathrm{D}_{2} \mathrm{O}$ solvent. The peak at $\delta=3.81$ was assigned to the superimposition of the methylene hydrogens, $a$ and $b$ ) joined with oxygen. The peak at $\delta=3.35$ was the methylene hydrogen joined with nitrogen (c). The peak at $\delta=1.35$ may be assigned to the methyl of product 2 , which didn't completely react in step 3 . 
Figure 4. ${ }^{1} \mathrm{H}-\mathrm{NMR}$ spectrum of phosphorus-containing triazine oligomer PMPT.

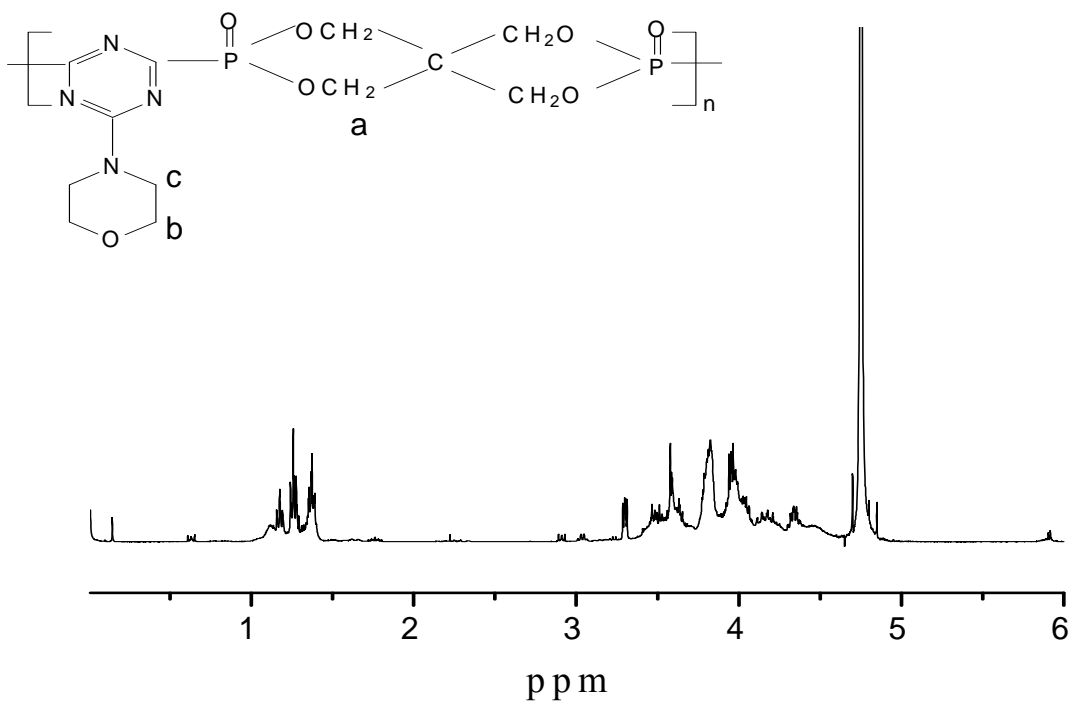

\section{3. $P$ NMR of PMPT}

Figure 5 shows the ${ }^{31} \mathrm{P}-\mathrm{NMR}$ spectrum of PMPT. The atoms and groups surrounding the phosphorus atom generally affect its chemical shift, especially for the atoms in the four chemical bonds surrounding the central phosphorus atom [12].

Figure 5. ${ }^{31} \mathrm{P}-\mathrm{NMR}$ spectrum of phosphorus-containing triazine oligomer PMPT.

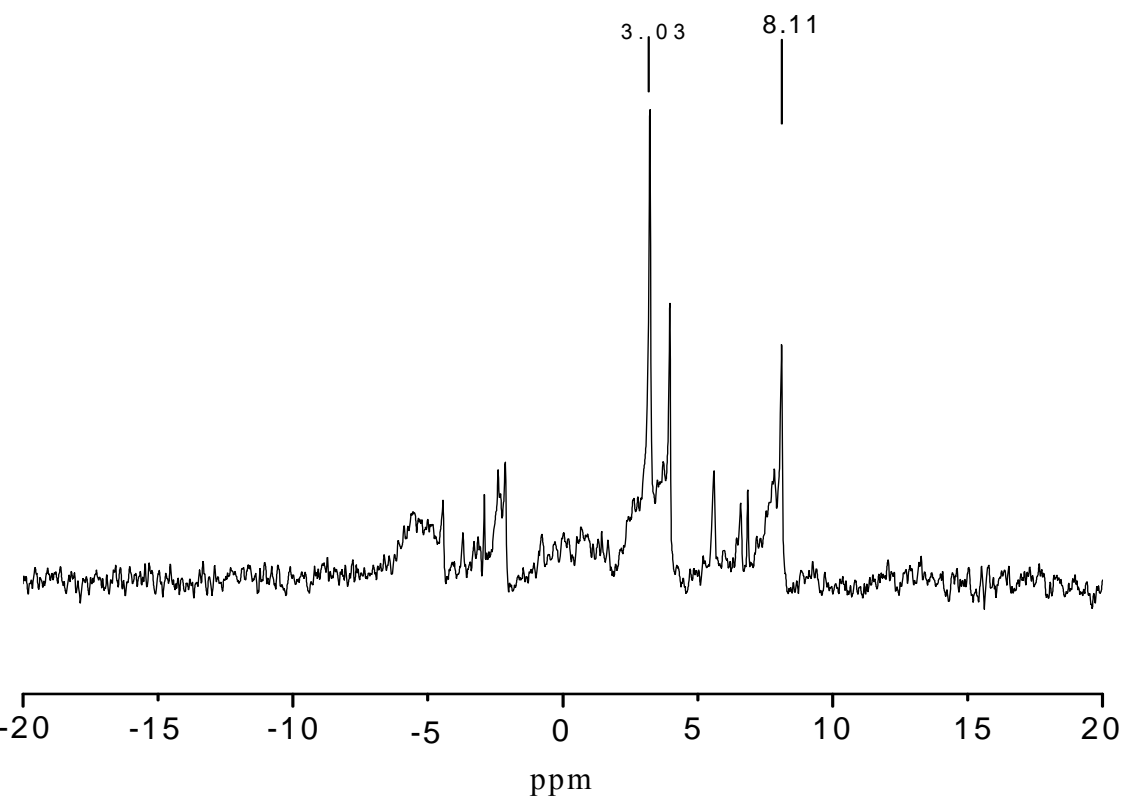

Figure 6 shows the presence of two kinds of phosphorus atoms. The stronger absorption at 3.03 ppm was assigned to the shift of $\mathrm{P}=\mathrm{O}$ group in product $\mathrm{A}$ [13]. The peak at $8.11 \mathrm{ppm}$ was the chemical shift of phosphorus in material B. The donating electron ability of the groups decrease with the distance of the three chemical bonds surrounding the phosphor atom in material B, compared with that of phosphorus in material $\mathrm{A}$, and the shielding effect of phosphorus atom on the external magnetic 
field becomes weaker, therefore the chemical shift of phosphorus in B is shifted to the low field. The ${ }^{1} \mathrm{H}-\mathrm{NMR}$ and ${ }^{31} \mathrm{P}-\mathrm{NMR}$ spectra are consistent with the chemical structures in Scheme 4.

Figure 6. Phosphorus atoms in two different chemical environments.

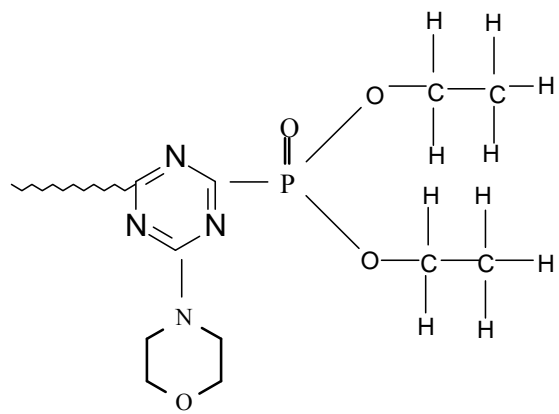

A

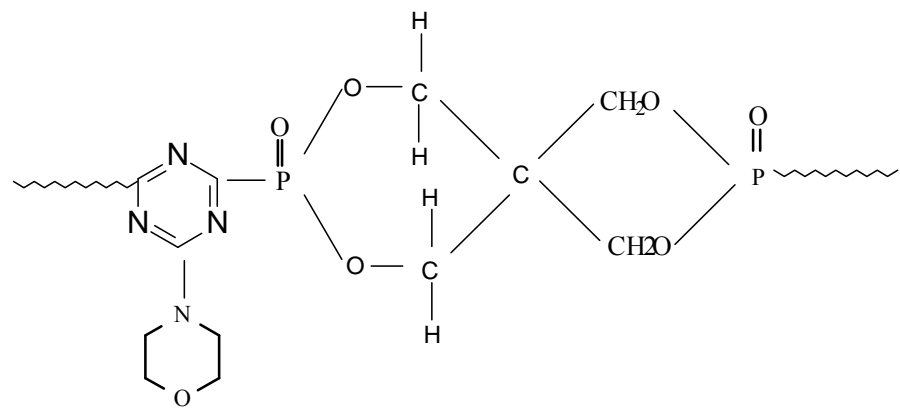

B

\subsection{Effect of PMPT and APP/PMPT on the flammability of FR-PP}

Table 1 shows effects of PMPT and APP/PER/PMPT on the LOI values of FR-PP. The LOI values of FR-PP improved with the increasing of PMPT content. When the content of PMPT is $30 \mathrm{wt} \%$, the LOI value of FR-PP increased about 59.7\% compared with that of PP, while it can achieve UL-94 V-0 rating. The flammability of PMPT is better than PPMT made by the former procedure because PPMT hasn't the sufficient carbon source and must be used together with APP, PER. Moreover the LOI value is slight higher than that of FR-PP with the same content of APP/PER. The LOI value can reach 29.4 after PMPT cooperates with APP/PER, which suggests that there is the synergistic effect between PMPT and APP/PER.

Table 1. Effect of PMPT and APP/PER/PMPT on the LOI.

\begin{tabular}{lcc}
\hline \multicolumn{1}{c}{ Sample } & LOI/\% & UL94 \\
\hline Pure PP & 18.1 & $\mathrm{HB}$ \\
$10 \%$ PMPT & 23.4 & $\mathrm{~V}-2$ \\
$30 \% \mathrm{PMPT}$ & 28.9 & $\mathrm{~V}-0$ \\
$30 \%$ APP/PER (3/1) & 27.8 & $\mathrm{~V}-0$ \\
$30 \%$ APP/PER/PMPT (3/1/2) & 29.4 & $\mathrm{~V}-0$ \\
\hline
\end{tabular}

\subsection{Effect of PMPT on the mechanical property of FR-PP}

Conventional IFRs always impair the mechanical properties of FR-PP because of the low thermal stability and the poor compatibility. Table 2 lists the mechanical properties of FR-PP with the different flame retardants. Though the mechanical properties decrease slightly with the increase of the PMPT content, the tensile strength and notched impact strength of FR-PP with 30\% PMPT increases about $31 \%$ and $23 \%$ more than those of FR-PP with the same content of APP/PER. The main molecular chain of PMPT contains carbon atoms, therefore the compatibility of PMPT and PP is better than that of other IFRs. 
Table 2. The mechanical properties of FR-PP with the different flame retardants.

\begin{tabular}{lcc}
\hline \multicolumn{1}{c}{ Sample } & Tensile strength (MPa) & Notched impact strength /J.m-1 \\
\hline Pure PP & 33.1 & 41.2 \\
10\%PMPT & 32.8 & 39.3 \\
30\%PMPT & 28.8 & 33.8 \\
30\%APP/PER (3/1) & 21.9 & 27.3 \\
30\%APP/PER/PMPT (3/1/2) & 23.4 & 29.6 \\
\hline
\end{tabular}

\section{Experimental}

\subsection{General}

IR spectra were recorded with a Vector-33 FTIR spectrometer using $\mathrm{KBr}$ pellets. ${ }^{1} \mathrm{H}-\mathrm{NMR}$ and ${ }^{13} \mathrm{P}$ NMR spectra $\left(\mathrm{D}_{2} \mathrm{O}\right.$ as solvent) were recorded on a Bruker Avance Digital spectrometer (operating at $400 \mathrm{MHz}$ for ${ }^{1} \mathrm{H}$ and ${ }^{13} \mathrm{P}$ ). The limited oxygen index (LOI) values were measured on a FTT oxygen index meter (England Fire Testing Technology Co., Ltd) with sheet dimensions of $130 \times 6.5 \times 3 \mathrm{~mm}$ according to ISO 4589-1984. The vertical flammability test is measured according to UL-94 test using $150 \times 13 \times 3 \mathrm{~mm}^{3}$ specimens.

\subsection{Materials}

Triethyl phosphite was supplied by Shandong Jincheng Pharmaceutical \& Chemical Co., Ltd. Tricyanogen chloride was obtained from Sigma-Aldrich Co. (St Louis, MO, USA). Analytically pure pentaerythritol was purchased from Shanghai Kefeng Chemical Reagents Co., Ltd. Morpholine was obtained from Shanghai Nanxiang Reagents Co., Ltd. The other reagents were all analytical reagents. Ammonium polyphosphate (APP) was obtained from Shandong Linqin Fuyuan Chemical Co., Ltd. Polypropylene was a commercially available grain with a $0.8 \mathrm{~g} / 10 \mathrm{~min}$ melt flow rate and was purchased from Sinopec Yanshan Chemical Corporation..

\subsection{Methods}

First, in a $1 \mathrm{~L}$ four-necked glass flask, equipped with a stirrer, thermometer, pressure equalizing addition funnel and cooling bath, tricyanogen chloride $(46.1 \mathrm{~g})$ and methylbenzene $(250 \mathrm{~mL})$ were added. Then triethyl phosphite $(124.5 \mathrm{~g})$ was added in $1 \mathrm{~h}$ while the mixture was stirred. The reaction mixture was then heated up to $70{ }^{\circ} \mathrm{C}$ for $6 \mathrm{~h}$ until no more gas was generated. Yellow crystals could be obtained after the methyl benzene was evaporated from the mixture and it was cooled down to roomtemperature. The crystals were filtered and recrystallized from ethyl ether. The white crystals of 2,4,6tri(diethyl phosphate)-1,3,5-triazine (1) weighed $105.4 \mathrm{~g}$ (yield: 86.1\%). The preparation of compound 1 was shown in Scheme 2. 
Scheme 2. Synthesis of compound 1.<smiles>Clc1nc(Cl)nc(Cl)n1</smiles><smiles>CCO[Pb](OCC)(OCC)C(C)(C)C</smiles><smiles>CCOP(=O)(OCC)c1nc(P(=O)(OCC)OCC)nc(P(=O)(OCC)OCC)n1</smiles>

Second, in a $1 \mathrm{~L}$ four-necked glass flask, equipped with a stirrer, thermometer, pressure equalizing addition funnel and cooling bath, compound 1 (97.8 g) and absolute alcohol (500 mL) were added. Morpholine (17.4 g) was then added and the mixture stirred for about $3 \mathrm{~h}$ at room temperature. Then the alcohol was evaporated from the mixture and the residue were washed by $n$-hexane and ethyl ether compound $(300 \mathrm{~mL}$, volume ratio: $1: 4)$ and the filtrate was concentrated at room temperature. The white crystalline product, 2-morpholinyl-4,6-di(diethyl phosphate)-1,3,5-triazine (2) weighed $81.1 \mathrm{~g}$ (yield: $92.5 \%$ ). The reaction is shown in Scheme 3.

Scheme 3. Synthesis of compound 2.

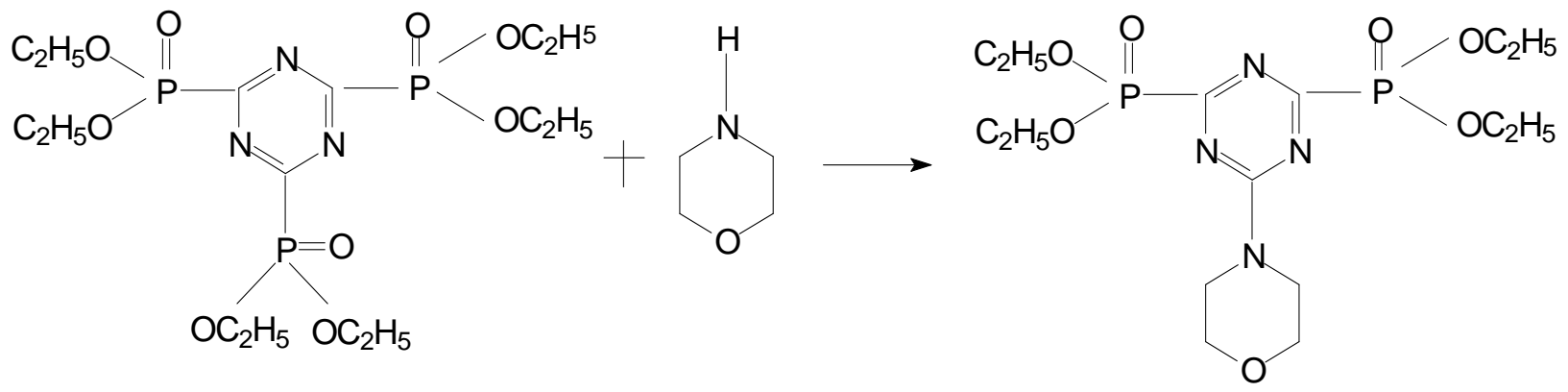

Third, in a $500 \mathrm{~mL}$ three-necked glass flask, equipped with a stirrer, thermometer and heating bath, compound 2 (43.8 g), pentaerythritol (13.6 g), dibutyl tin oxide catalyst (1.0 g) and N,N-dimethylformamide (DMF, $200 \mathrm{~mL}$ ) were added.

Scheme 4. Synthesis of PMPT.

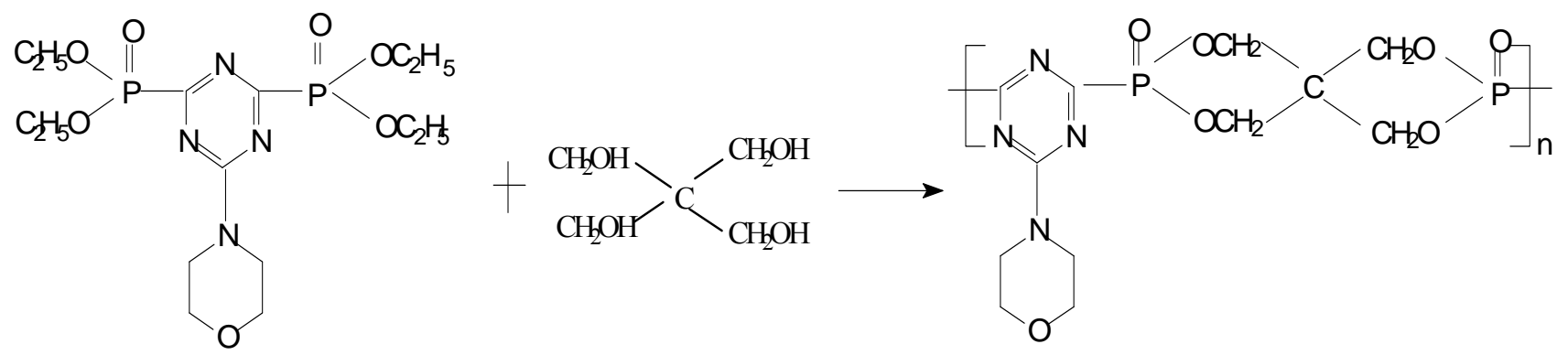

The mixture was stirred for $24 \mathrm{~h}$ at $130{ }^{\circ} \mathrm{C}$ and the alcohol was evaporated continually. After this, a yellow solid was obtained after the DMF was distilled off. The white crystalline phosphorus- 
containing triazine oligomer poly(2-morpholinyl-4-pentaerythritol phosphate-1,3,5-triazine) (PMPT, 3) weighed $49.1 \mathrm{~g}$ (yield: 93.6\%) was the after the yellow solid was recrystallized from tetrahydrofuran and washed with alcohol.

\section{Conclusions}

The phosphorus-containing triazine oligomer PMPT was synthesized by a three step chemical reaction. The band at $1,261 \mathrm{~cm}^{-1}$ in the FTIR and attributed to the $\mathrm{P}=\mathrm{O}$ group stretching vibration indicated that the chemical reaction between triethyl phosphite and tricyanogen chloride had occurred. The absorption signal at $1,116 \mathrm{~cm}^{-1}$ was attributed to the asymmetric stretching vibration of the ether link on the morpholinyl ring. ${ }^{1} \mathrm{H}-\mathrm{NMR}$ and ${ }^{31} \mathrm{P}-\mathrm{NMR}$ spectra further confirmed the chemical structure of PMPT. The slight effect of PMPT on the mechanical properties of FR-PP suggested that the compatibility of PMPT and PP is better than other IFRs. The high LOI values of FR-PP revealed that PMPT is an efficient IFR and there is the synergistic effect between PMPT and APP/PER.

\section{Acknowledgements}

This project was supported by the Shenzhen Science and Technology Research Grant (CXB200903090012A).

\section{References and Notes}

1. Pal, K.; Rastogi, J.N. Development of halogen-free flame-retardant thermoplastic elastomer polymer blend. J. Appl. Polym. Sci. 2004, 94, 407-415.

2. Ma, H.Y.; Tong, L.F.; Xu, Z.B.; Fang, Z.P.; Jin, Y.M.; Lu, F.Z. Charge disproportionation in quasi-one-dimensional vanadium oxides. Polym. Degrad. Stab. 2007, 92, 720-726.

3. Gao, F.; Tong, L.F.; Fang, Z.P. Some blow-up problems for a semilinear parabolic equation with a potential. Polym. Degrad. Stab. 2006, 91, 1295-1299.

4. Wang, H.F.; Zhu, P.; Zhang, J.B.; Wang, B. Preparation and application of triazinyl phosphorus flame retardant for cotton fabrics, Textile Auxiliar. 2005, 9, 15-17.

5. Hu, X.P.; Li, Y.L.; Wang, Y.Z. Synergistic Effect of the Charring Agent on the Thermal and Flame Retardant Properties of Polyethylene. Macromol. Mater. Eng. 2004, 289, 208-212.

6. Heinrich, H.; Stefan, P. The importance of intumescent systems for fire protection of plastic materials, Polym. Int. 2000, 49, 1106 1114;

7. Chiu, S.H.; Wang, W.K. The dynamic flammability and toxicity of magnesium hydroxide filled intumescent fire retardant polypropylene. J. Appl. Polym. Sci 1998, 67, 989-995.

8. Liu, G.; Zhao, J.Q.; Zhang, Y.H.; Liu, S.M.; Ye, H. Synthesis and Application in Polypropylene of a Novel Nitrogen-Containing Intumescent Flame Retardant. Polym. Compos. 2007, 15, 191-198.

9. Benjaporn, T.; Chitchamai, O.; Juraithip, W.; Ruth, D.; Ruedeekorn, W. Effect of PEG molecular weight and linking chemistry on the biological activity and thermal stability of PEGylated trypsin. Int. J. Pharm. 2008, 357, 252-259. 
10. Gang, K.; Homogeneous modification of carbon nanotubeswith cellulose acetate, Chin. Chem. Lett. 2009, 20, 1376-1380.

11. Wong, S.P. Fourier Transform Infrared Spectrometer, 2nd ed.; Chemical Industry Press: Beijing, China, 2005; pp. 239-247.

12. Tong, J.B.; Zeng, H.; Zhang, S.W.; Zhou, P.; Yang, S.X.; Feng, Y.L.; Zhang, Q.X.; Li, Z.L. Prediction of the $\mathrm{P}$ chemical shifts in nuclear magnetic resonance spectroscopy for 291 phosphines. Chin. J. Anal. Chem. 2006, 34, 1007-1010.

13. Liu, G.; Liu, S.M.; Sheng, Q.; Zhao, J.Q.; Zhang, Y.H.; Zhang, Z.H. Synthesis and characterization of phosphorus-containing triazine polymer, Petrochem. Technol. 2007, 36, 173-177.

Sample Availability: Samples of the compounds 1,2 and PMPT are available from authors.

(C) 2010 by the authors; licensee MDPI, Basel, Switzerland. This article is an open access article distributed under the terms and conditions of the Creative Commons Attribution license (http://creativecommons.org/licenses/by/3.0/). 